\title{
Spin mixing processes in magnetic nanostructures detected by thermoelectric measurements
}

\author{
L. Gravier ${ }^{1}$, S. Serrano Guisan ${ }^{1}$, G. di Domenicantionio ${ }^{2}$, M. Abid ${ }^{1}$, M. \\ Hillenkamp $^{2}$, C. FÉlix ${ }^{2}$ and J.-Ph. Ansermet ${ }^{1}$ \\ 1 Laboratoire de Physique des Matériaux Nanostructurés, \\ 2 Laboratoire des Nanostructures en Matrices, \\ Ecole Polytechnique Fédérale de Lausanne, CH-1015 Lausanne-EPFL, Switzerland
}

PACS. 72.25. Ba - Spin polarized transport in metals.

PACS. 75.75.+a - Magnetic properties of nanostructures.

PACS. 81.07.-b - Nanoscale materials and structures: fabrication and characterization.

\begin{abstract}
Version: 3.11.2006
Abstract. - Spin-dependent transport properties of magnetic nanostructures have been investigated by means of magneto-thermogalvanic voltage measurements: the ac voltage response to an ac temperature oscillation is measured for various magnetic nanostructures under dc current. The samples studied include $\mathrm{Co} / \mathrm{Cu}$ multilayered nanowires, homogeneous Ni nanowires and cobalt clusters embedded in copper films. The magnetic field dependence of this signal is always larger than the magneto-resistance (MR) and may be anisotropic even when the MR is not. A thermodynamic argument introduces spin mixing as the main process measured by this novel thermoelectric measurement technique. This effect is not observed in magnetite as can be justified by the absence of an accessible second spin channel.
\end{abstract}

Spin-dependent transport in magnetic materials is one of the key ingredients of spintronics and has already demonstrated its importance in effects like Giant magnetoresistance (GMR) [1,2]. Valet and Fert described this effect with the help of a two-current model [3], neglecting the reduction of GMR due to spin mixing processes, on the basis that they were negligible. In the present paper, we investigate these spin mixing effects by means of a novel thermoelectric experiment, called magneto-thermogalvanic voltage (MTGV). We have applied this measurement technique to various magnetic systems, like nanowires (homogenous and multilayered) and films (granular structures). Our data show that the observed magnetic field response must arise from a mechanism distinct from GMR. A thermodynamic approach, based on transport matrix for a magnetic medium [4], suggests that MTGV depends on spin mixing effects.

Nanowire samples are obtained by an electrochemical technique of template synthesis. Pores of track-etched polymer membranes are filled by means of electrodeposition. Multilayer nanowires are composed of a series of 300 bilayers of $\mathrm{Co}$ and $\mathrm{Cu}, 10 \mathrm{~nm}$ each. They are typically $6 \mu \mathrm{m}$ long with a diameter of 30 to $60 \mathrm{~nm}$ and are electrically contacted to $\mathrm{Au}$ layers on both side of the membrane (fig. 1.a). By monitoring the potential between the (c) EDP Sciences 

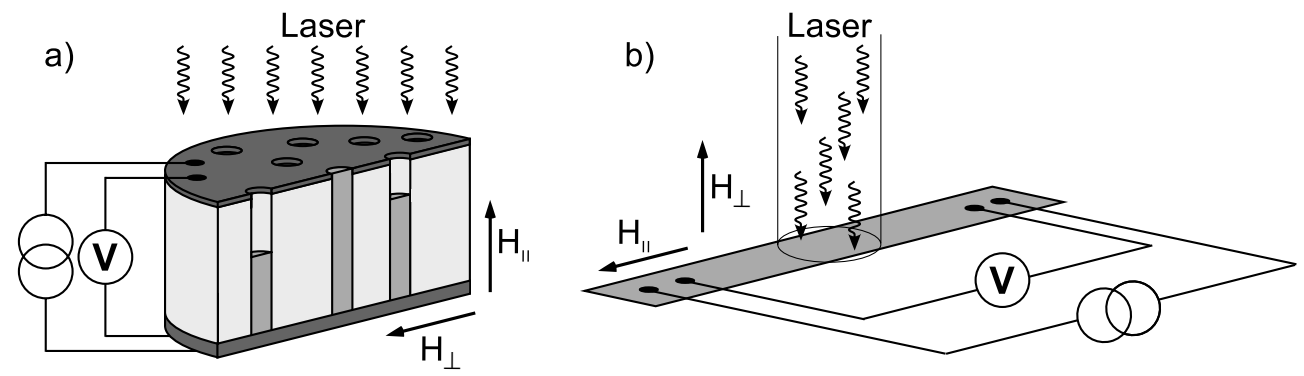

Fig. 1 - Schematic pictures of the investigated structures. a) Nanowires (grey) embedded in a polymer membrane (light grey). A single nanowire is electrically contacted to the gold electrodes (dark grey). b) Thin film of granular material. The laser spot illuminates only one portion of the stripe. Thick arrows denote the orientations of the applied magnetic field, parallel $(\|)$ or perpendicular $(\perp)$ to the wire axis or the film plane.

two $\mathrm{Au}$ electrodes we can stop the deposition as soon as the contact to a single nanowire is established [5].

Granular film samples are prepared according to the strategy of "cluster-assembled materials" [6]. The samples consist of thin films of copper in which are dispersed cobalt clusters of well-defined size. Narrow distributions of metal cluster ions are prepared and analyzed in the gas phase and co-deposited with the copper matrix on a polyimide substrate. The film thickness and Co loading were $50 \mathrm{~nm}$ and $8 \%$, respectively. The mean size of embedded clusters used in this study was 15 atoms per cluster. We performed our measurements on stripes of 2 x $20 \mathrm{~mm}$, connected to the electrodes with silver paste (fig. 1.b).

Besides magneto-resistance (MR), we measured the thermogalvanic voltage (TGV) as follows. A chopped laser diode irradiates the sample and induces a temperature oscillation of about $1 \mathrm{~K}$. The corresponding voltage across the sample is measured by lock-in detection under a constant charge current $I_{d c}$. Consequently, TGV measurements probe the first derivative of the effective conductivity with respect to temperature. It has been shown in the case of multilayers that the major contribution to the voltage arises from thermoelectric processes, e.g. Peltier-like effects [7]. There is also a contribution from the derivative of the resistance with respect to temperature, but it is not essential for the present argument: it is small and even negligible at low temperature, and shows no magnetic field dependence. Its magnitude in the particular case of magnetite is discussed separately at the end of this paper.

The laser induces an oscillating temperature gradient in the nanowire samples. Via the Seebeck effect, the oscillating temperature gradient contributes to the TGV. However, this spurious effect is negligible, being in the microvolt range while the TGV is in the millivolt range typically [8]. Experiments on the granular samples were performed with the laser spot centered between the electrodes so as to suppress any contribution of the thermoelectrical power.

Results of MR and MTGV measurements performed on three different representative systems are presented in fig. 2. For all systems, the MTGV differs strongly qualitatively and quantitatively from the MR, indicating that these experiments detect different underlying processes.

- In Co-Cu multilayers (fig. 2.a), GMR is independent of the field orientation, the field dependence is a bell-shaped curve. The MTGV shows a large positive variation and, unlike 

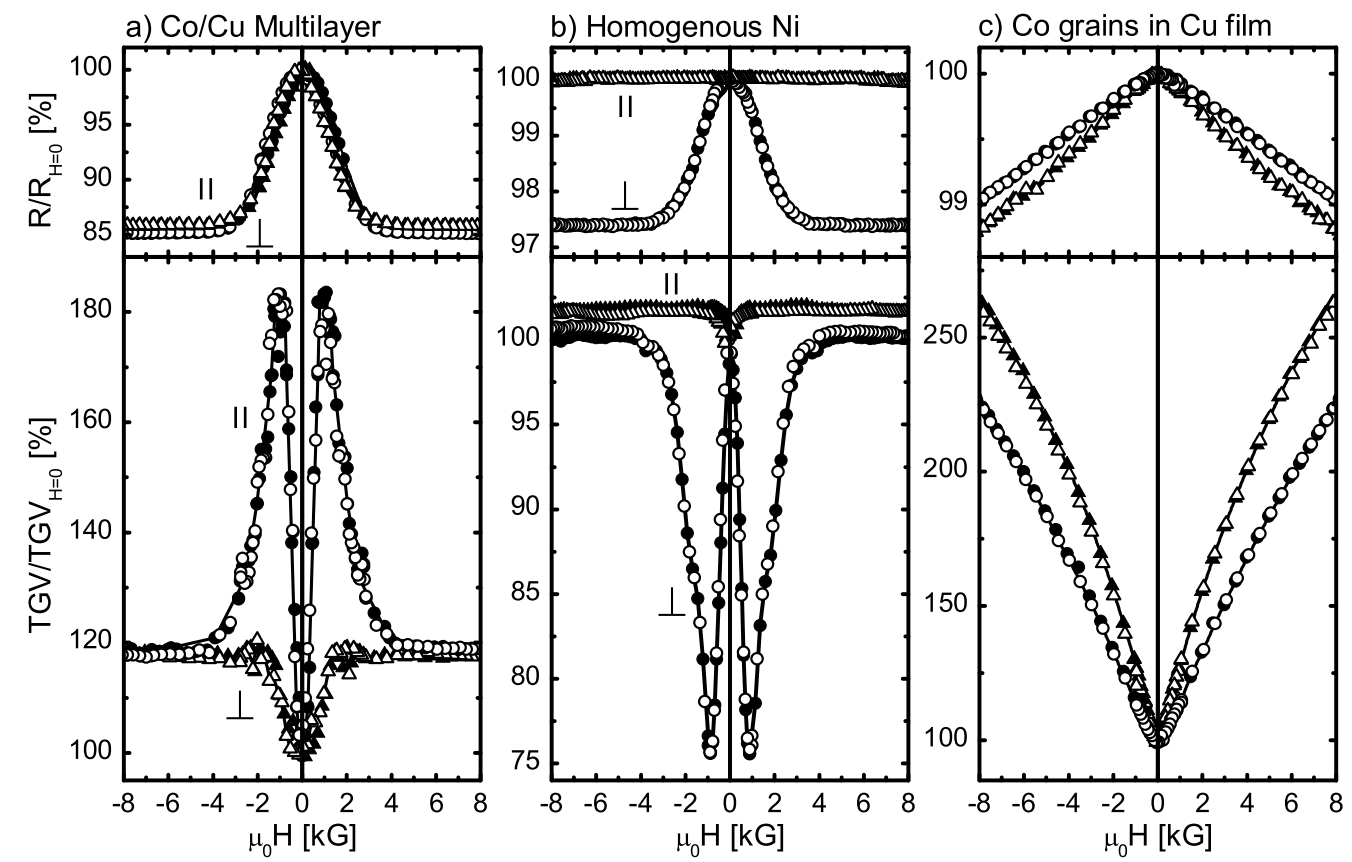

Fig. 2 - MR and MTGV curves normalized to their zero-field value for different systems. a) Co-Cu multilayered nanowire at 300K. b) Homogenous Ni nanowire at 300K. c) Granular film of Co clusters $(\langle n\rangle=15)$ in a $\mathrm{Cu}$ matrix at $14 \mathrm{~K}$. Circles and triangles denote the external magnetic field respectively perpendicular $(\perp)$ and parallel $(\|)$ to the wire axis or the thin film plane. Solid and open symbols show positive and negative field sweeps, respectively.

GMR, a strong anisotropy: for the external magnetic field applied along the wire axis, i.e. normal to the layers, the MTGV is not monotonic. The largest variation of $80 \%$ corresponds to the steepest slope of the magnetoresistive curve, that is, to the most unstable configuration of the magnetic moments of each layer, forced out-of-plane in this case [8]. This feature is especially obvious for nanowire systems composed of a limited number of layers, as shown in reference [9].

- In homogenous Nickel nanowires (fig. 2.b), an anisotropic magnetoresistance (AMR) of $2.5 \%$ is observed [10]. The MTGV also shows an anisotropy, but in a very different way: a perpendicular magnetic field results in a decrease of $25 \%$, ten times larger than AMR, and a non-monotonic field dependence. Here again, the largest amplitude of the MTGV corresponds to the maximum in slope of the AMR curve. At this point the magnetization is very unstable [11] and is forced by the external field to move from the wire axis towards the transverse direction. This is also observed in the jump in magnetization for the magnetic field off the perpendicular direction $\left(80^{\circ}\right.$ with respect to the wire axis $)$ as shown in fig. 3 . This switching results in a jump of about $0.1 \%$ in resistance only, whereas a clear jump $>1 \%$ is measured in 


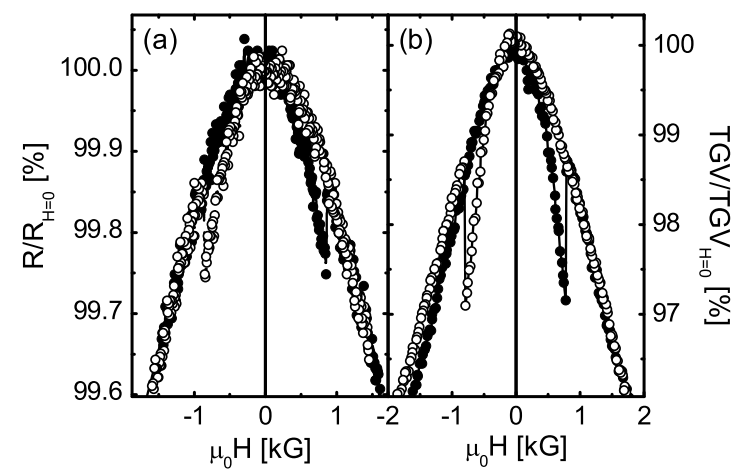

Fig. 3

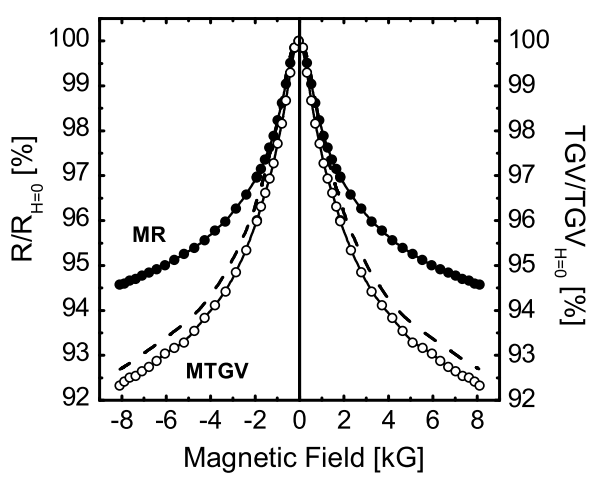

Fig. 4

Fig. 3 - MR (a) and MTGV (b) curves for a Nickel nanowire measured at 300K for an angle of $80^{\circ}$ between wire axis and external magnetic field $\mathrm{H}$. The jumps show where the magnetization switches along H. Solid and open symbols show positive and negative field sweep, respectively.

Fig. 4 - MR(solid circles) and MTGV (open circles) curves for a $\mathrm{Fe}_{3} \mathrm{O}_{4}$-nanowire. The dashed line shows the calculated ohmic contribution to the TGV signal.

the TGV. The highly unstable magnetization state preceding the jump results in a maximum variation of MTGV.

- In the granular films, a magnetoresistance of $\sim 1 \%$ at $0.8 \mathrm{~T}$ is found. Although saturation is not reached, the order of magnitude agrees with the literature on granular systems [12]. The MTGV shows a field dependence of opposite sign and two orders of magnitude larger, about $265 \%$ at $0.8 \mathrm{~T}$ for the present sample [13]. Both MR and MTGV curves are monotonic for this type of sample. While the MR of granular samples only varies little between $15 \mathrm{~K}$ and 300K, their MTGV completely vanishes at room temperature.

Since the temperature and field dependence of the MTGV is qualitatively so different from that of the MR, it is clear that MTGV detects a spin-dependent effect completely different from MR and highly sensitive to the magnetic configuration of the system. We will now suggest, by means of a thermodynamic argument, that the basic origin of this mechanism arises from spin mixing processes. The formalism of thermodynamics of irreversible processes in the linear approximation [14] allows us to describe the thermodynamic transport in terms of an Onsager matrix [4]. We state a linear dependence of current densities of entropy $j_{s}$ and of charges $j_{\uparrow}$ and $j_{\downarrow}$ on the generalized forces $\nabla T$ and $\nabla \bar{\mu}_{\uparrow, \downarrow}$, where $\bar{\mu}_{\uparrow, \downarrow}$ are the electrochemical potentials of the electrons of each spin orientation $\uparrow$ and $\downarrow[3]$.

$$
\left(\begin{array}{c}
\mathbf{j}_{s} \\
\mathbf{j}_{\uparrow} \\
\mathbf{j}_{\downarrow}
\end{array}\right)=-\left(\begin{array}{ccc}
L_{s s} & L_{s \uparrow} & L_{s \downarrow} \\
L_{\uparrow s} & L_{\uparrow \uparrow} & L_{\uparrow \downarrow} \\
L_{\downarrow s} & L_{\downarrow \uparrow} & L_{\downarrow \downarrow}
\end{array}\right)\left(\begin{array}{c}
\nabla T \\
\nabla \bar{\mu}_{\uparrow} \\
\nabla \bar{\mu}_{\downarrow}
\end{array}\right) .
$$

The parameters $L_{\uparrow \uparrow}$ and $L_{\downarrow \downarrow}$ account for the usual spin polarized conductivities revealed in MR measurements. The novelty in eq. (1), compared to e.g. the formalism of Valet and 
Fert [3], is to introduce the off-diagonal terms $L_{\uparrow \downarrow}$ and $L_{\downarrow \uparrow}$, that express the feeding of one spin current under the effect of the gradient of potential of the other spin channel. In this sense, $L_{\uparrow \downarrow}$ and $L_{\downarrow \uparrow}$ describe spin mixing.

The transport matrix in eq. (1) was exploited in one case so far: magnetic multilayers with current perpendicular to the planes in the limit where the layers are thin enough that the gradients of temperature and of electrical potential can be assumed constant over a layer thickness [4]. This approach cannot be applied to granular systems, the geometry of the current path being much too complicated. In place, an analysis in terms of an effective medium was attempted as a first approximation just for the sake of expliciting the potential role of spin mixing in transport in this case [15].

Without the spin mixing terms $L_{\uparrow \downarrow}$ and $L_{\downarrow \uparrow}$, the magnetic field dependence of the transport would be carried by the spin dependent conductivities $L_{\uparrow \uparrow}$ and $L_{\downarrow \downarrow}$. This would imply that MTGV should be similar to MR, in both shape and amplitude. As the data show clear distinctions between MR and MTGV, we conclude that the MTGV signal results form the spin mixing terms. Whereas conductivities $L_{\uparrow \uparrow}$ and $L_{\downarrow \downarrow}$ naturally dominate in resistance measurements, the TGV measurement based on temperature oscillations strongly favors the thermoelectric voltages dominated by spin mixing terms [8].

The main spin mixing mechanism usually invoked for ferromagnetic materials is the scattering of electrons with long wavelength magnons, more specifically the asymmetry between spin up-to-down and down-to-up scattering rates [16]. This is likely to happen in ferromagnetic nanowire structures sufficiently large for spin waves to develop. The efficiency of this mechanism was determined to be much stronger in Nickel than in Cobalt [17]. We have indeed found almost zero MTGV in homogenous Co nanowires prepared and measured identically (not shown here). This mechanism is naturally invoked in the case of $\mathrm{Co}-\mathrm{Cu}$ multilayer nanowire systems [4], but is apparently assisted by additional spin mixing processes as suggested by the non-monotonic behaviour of MTGV in parallel fields (fig. 2.a) [8,9]. A thermokinetic approach identifies this anisotropy, also observed by magneto-thermoelectrical power measurements, to interface spin mixing effects [18].

Spin mixing may also occur when an electron crosses a magnetic domain wall. This is simply the result of a spin-dependent scattering probability, the spin not quite tracking the exchange field as the electron crosses the domain wall, which is equivalent to inducing spin transitions [19]. This may also explain the small MTGV peak at zero field observed for parallel fields (fig. 2.b) and the large MTGV response just before the magnetization reversal in $\mathrm{Ni}$ nanowires (fig. 3.a).

In the case of granular systems, long wavelength spin waves cannot develop in the small grains of $\sim 15$ Co atoms [15]. Another spin mixing mechanism denominated "jitterbug" has been described in the literature [20]. Here the conduction electron's spin is assumed to precess about the exchange field inside the magnetic grain as it traverses it, thus causing transitions between the two spin states [21]. An external magnetic field orients the granular magnetic moments and thus induces a spin asymmetry of this process.

The idea that spin mixing is crucial in MTGV measurement is further supported by the study of $\mathrm{Fe}_{3} \mathrm{O}_{4}$ (magnetite) nanowires [22]. This is considered as a typical half-metallic material, in which only one single spin channel is allowed at the Fermi energy [23]. As a consequence, no spin mixing is theoretically possible, although it has been pointed out that such a process may nevertheless occur at grain boundaries [24]. Our measurements in Fig. 4 show a MR of about $9 \%$ is typical for the inhomogeneous microcrystalline structure. The dashed line corresponds to the contribution of the MR to the TGV signal, deduced from the temperature dependence of the resistance. It accounts for about $95 \%$ of the measured MTGV. This 
confirms the very small contribution of spin mixing, as expected in this half-metallic medium.

In summary, we performed a special kind of thermoelectric measurement which we call MTGV, on various magnetic nanostructures. The large differences in field response as compared to magnetoresistance demonstrate that the MTGV detects spin dependent transport processes different from those involved in regular MR measurements. Using a simple thermodynamic argument, we propose spin mixing as the leading mechanism responsible for the field sensitivity of our MTGV experiments. Electron-magnon scattering and spin precession about the exchange field are the spin mixing mechanisms considered for nanowire and granular systems. This analysis is further supported by the comparative study of absence of spin mixing effect detected in half-metallic magnetite nanowires.

\section{REFERENCES}

[1] Baibich M. N., Broto J. M., Fert A., Nguyen Van Dau F., Petroff F., Etienne P., Creuzet G., Friedrich A. and Chazelas, J., Phys. Rev. Lett., 61 (1988) 2472.

[2] Binasch G., Grünberg P., Saurenbach F. and Zinn W., Phys. Rev. B, 39 (1989) 4828.

[3] Valet T. and Fert A., Phys. Rev. B, 48 (1993) 7099.

[4] Gravier L., Serrano-Guisan S., Reuse F. and Ansermet J.-Ph., Phys. Rev. B, 73 (2006) 024419.

[5] Wegrowe J.-E., Gilbert S. E., Kelly D., Doudin B. and Ansermet J.-Ph., IEeE Trans. Magn., 34 (1998) 903.

[6] Hillenkamp M., di Domenicantonio G. and Félix C., Rev. Sci. Instrum., 77 (2006) 25104.

[7] Gravier L., Serrano-Guisan S. and Ansermet J.-Ph., J. Appl. Phys., 97 (2005) 10C501.

[8] Gravier L., Serrano-Guisan S. and Ansermet J.-Ph., Phys. Rev. B, 73 (2006) 0521410.

[9] Serrano-Guisan S., Gravier L., Abid M. and Ansermet J.-Ph., Mat. Sci. Eng. B, 126 (2006) 292.

[10] Jaccard Y., Guittienne Ph., Kelly D., Wegrowe J.-E. and Ansermet J.-Ph. , Phys. Rev. B, 62 (2000) 1141.

[11] Wegrowe J.-E., Meier J. P., Doudin b., Ansermet J.-Ph., Wernsdorfer W., Barbara B., Coffey W. T., Kalmykov Y. P. And DéJardin J.-L., Europhys. Lett., 38 (1997) 329.

[12] Parent F., Tuaillon J., Stern L. B., Dupuis V., Prevel B., Perez A., Melinon P., Guiraud G., Morel R., Barthélémy A. and Fert A., Phys. Rev. B, 55 (1997) 3683.

[13] Measurements performed on several granular samples show a MTGV ranging from $150 \%$ to $500 \%$ [15]. This variation is attributed to local inhomogeneities of the cluster density.

[14] Callen H. B., Thermodynamics (John Wiley \& Sons, New York) 1960

[15] Serrano-Guisan S., di Domenicantonio G., Abid M., Abid J.-P., Hillenkamp M., Gravier L., Ansermet J.-Ph. and Félix C., Nature Materials, 5 (2006) 730.

[16] Piraux L., Fert A., Schroeder P. A., Loloee R. and Etienne P., J. Magn. Magn. Mat., 110 (1992) L247.

[17] Raquet B., Viret M., Sondergard E., Cespedes O. and Mamy R., Phys. Rev. B, 66 (2002) 024433.

[18] Wegrowe J.-E., Ahn Nguyen Q., Al-Barki M., Dayen J.-F., Wade T. L. and Drouhin H.-J., Phys. Rev. B, 73 (2006) 134422.

[19] Marrows C. H. and Dalton B. C., Phys. Rev. Lett., 92 (2004) 097206.

[20] Gregg J. F., Allen W., Thompson S. M., Watson M. L. and Gehring G. A., J. Appl. Phys., 79 (1996) 5593.

[21] Gehring G. A., Gregg J. F., Thompson S. M. and Watson M. L., J. Magn. Magn. Mater., 140-144 (1995) 501.

[22] Terrier C., Abid M., Arm C., Serrano-Guisan S., Gravier L. and Ansermet J.-Ph., J. Appl. Phys., 98 (2005) 086102. 
L. Gravier, S. Serrano Guisan, G. Di Domenicantionio, M. Abid, M. Hillenkamp, C. Félix and J.-Ph. Ansermet: Spin mixin

[23] Coey J. M. D. and Chien C. L., MRS Bull., 28 (2003) 724.

[24] Dowben P. A. and Skomski R., J. Appl. Phys., 95 (2004) 7453. 